\title{
Further Phenotypic Delineation of Partial Trisomy 17q and Partial Monosomy 20q due to Rare $t(17 ; 20)$
}

\author{
Gizem Ürel-Demir ${ }^{a}$ Özlem Akgün-Doğan ${ }^{a}$ Sümeyra Oğuz ${ }^{b}$ \\ Naz Güleray-Lafcı ${ }^{b}$ Pelin Özlem Şimşek-Kiper ${ }^{a}$ Gülen Eda Utine ${ }^{a}$ \\ Mehmet Alikaşifoğlu ${ }^{b}$ Koray Boduroğlu ${ }^{a}$ \\ ${ }^{\mathrm{a}}$ Department of Pediatric Genetics, Faculty of Medicine, Hacettepe University Ankara, Turkey; ${ }^{\mathrm{b}}$ Department of \\ Medical Genetics, Faculty of Medicine, Hacettepe University, Ankara, Turkey
}

\section{Established Facts}

- Chromosome deletions or duplications may arise both de novo or inherited, while coexistence of a deletion and a duplication in a patient suggests unbalanced segregation of a balanced translocation in one of the parents.

\section{Novel Insights}

- Craniosynostosis is not an essential feature of partial trisomy $17 \mathrm{q}$ and concomitant partial monosomy 20q.

- Karyotype analysis is not always sufficient to expose balanced translocations in parents, when the segments are shorter than $5 \mathrm{Mb}$.

\section{Keywords}

Chromosome $17 q$ duplication . Chromosome $20 q$ deletion . Copy number variations · Intellectual disability · Microarray analysis

\section{Abstract \\ Copy number variations in subtelomeric regions of chromo- somes 17 and 20 are associated with intellectual disability and various systemic manifestations. Microarray analysis al- lows identification of submicroscopic chromosomal abnor- malities and is applicable to elucidate the etiology of cogni- tive impairment in approximately one-fifth of the cases. In the present study, we report on 3 male children from 2 sis-}

ters, who suffered from intellectual disability, facial dysmorphism, and epilepsy. Despite the initial suggestion of an Xlinked inheritance, the condition was associated with 17q25.3 duplication and concomitant 20q13.33 deletion, as detected by microarray analysis. Coexistence of a deletion and a duplication suggests unbalanced segregation of a parental balanced translocation. Further investigations revealed maternal balanced translocations, which resulted in copy number aberrations in the children following unbalanced segregations. The work-up underlined the importance of genomic screening using microarrays as the firsttier diagnostic tool in intellectual disability, despite an apparent X-linked segregation in the pedigree.

(c) 2020 S. Karger AG, Base

\section{KARGER}

(c) 2020 S. Karger AG, Basel

karger@karger.com

www.karger.com/msy
Gizem Ürel-Demir

Department of Pediatric Genetics

Faculty of Medicine, Hacettepe University

Sihhiye, Ankara 06100 (Turkey)

E-Mail gizemurel@gmail.com 
Fig. 1. Photographs of the patients showing dysmorphic facial features. Patients 1 (a), 2 (b), and 3 (c).
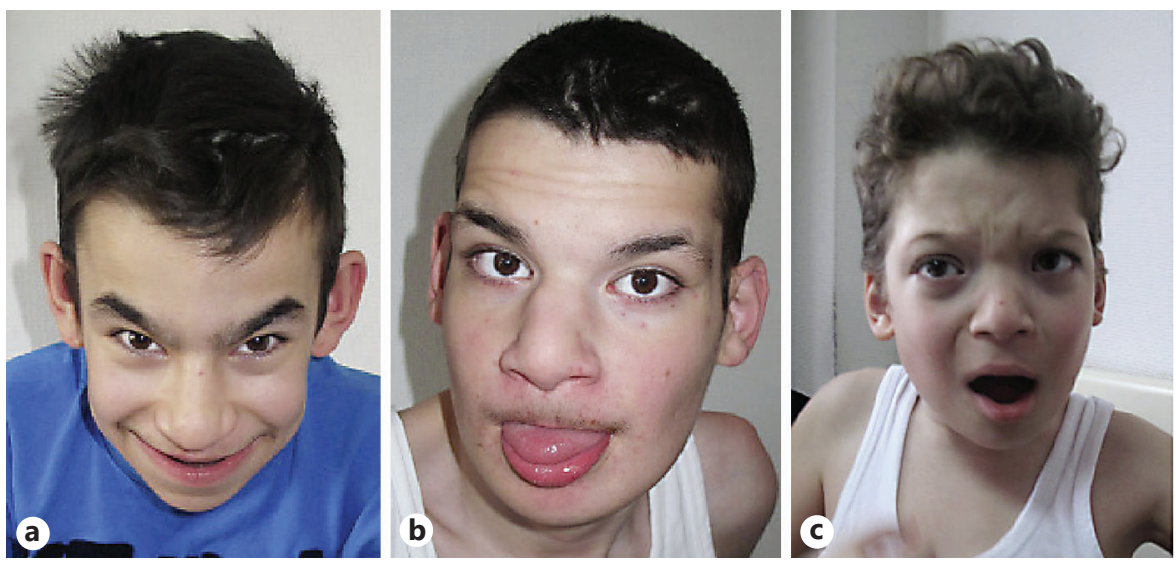

Clinical relevance of copy number variations (CNVs) in intellectual disability (ID) is well-established, for which CNVs constitute the leading genetic etiology. Genomewide CNVs have been described by the widespread use of microarray-based comparative genomic hybridization (aCGH).

Patients with $17 \mathrm{q}$ partial duplications exhibit a distinctive phenotype, including ID, facial dysmorphisms, and systemic congenital malformations [Upadia et al., 2018]. On the other hand, subtelomeric deletions of 20q present with ID, delayed speech, growth retardation, skeletal malformations, and seizures, without distinguishable facial dysmorphism [Traylor et al., 2010]. To the best of our knowledge, only 1 patient with combined duplication of $17 q 25.3$ and deletion of $20 \mathrm{q} 13.33$ has been reported to date [Marques et al., 2015]. Herein, we describe 3 patients from a family affected by partial $17 \mathrm{q} 25.3$ gain and $20 \mathrm{q} 13.33$ loss.

\section{Case Presentations}

\section{Patient 1}

A 12-year-old boy was referred to the pediatric genetics department because of ID and dysmorphic features. He was born at fullterm by normal vaginal delivery, with a birth weight of 2,800 g, to nonconsanguineous parents. He was diagnosed with epilepsy at the age of 13 days that remained in remission after 4 years and had developmental delay (DD). The boy underwent surgery for cryptorchidism. Physical examination revealed brachycephaly, a long face, prominent metopic suture, synophrys, anteverted ear lobes, macrostomia, a single palmar crease on the left hand, bilateral metacarpophalangeal joint hyperextensibility, and kyphosis (Fig. 1a). Stereotypic movements and happy demeanor were observed. Anthropometric measurements were in normal range. Previously assayed karyotype analysis, fragile $\mathrm{X}$ analysis, brain MRI, and abdominal ultrasonography were all normal. Mitral regurgita- tion was detected by echocardiography. The 2 sons of his maternal aunt, suffering from similar conditions, were invited to the clinic.

\section{Patient 2}

The 15-year-old cousin of the proband presented with ID. He was born at 36 weeks' gestation with a birth weight of 2,300 g, after an uneventful pregnancy. Parents denied consanguinity. The mother had cleft palate surgery. The patient had seizures starting at day 11 and global DD. Surgery was undertaken for cryptorchidism and strabismus. Clinical examination revealed microcephaly (head circumference: $52 \mathrm{~cm},-3.16 \mathrm{SD}$ ), double hair whorls, bitemporal narrowing, sparse lateral eyebrows, deep-set eyes, upslanting palpebral fissures, prominent nasal bridge and columella, thin upper lip, malocclusion, and anteverted ears (Fig. 1b). He also had pectus carinatum, scoliosis, and metacarpophalangeal laxity. Previous genetic investigations including karyotyping, fragile $\mathrm{X}$ analysis, and FISH for Angelman syndrome were normal. Brain MRI was unremarkable. Echocardiogram demonstrated mitral valve regurgitation.

\section{Patient 3}

The 9-year-old brother of patient 2 presented with DD and ID. Antenatal history was unremarkable. He had seizures starting the first week of life. Brain MRI was normal. Echocardiogram revealed peripheral pulmonary stenosis and secundum atrial septal defect. Renal ultrasound revealed unilateral pelviectasis. The G-banded karyotype was normal. On examination, his height was $121 \mathrm{~cm}$ (-2.07 SD), weight $26 \mathrm{~kg}(-0.69 \mathrm{SD})$, and head circumference 52 $\mathrm{cm}(-0.73 \mathrm{SD})$. He had a prominent forehead, hypertelorism, upslanting palpebral fissures, high-arched palate, overfolded helices, pectus carinatum, and pes planus (Fig. 1c).

\section{Materials and Methods}

\section{Array $\mathrm{CGH}$}

Genomic DNA was extracted from leukocytes of EDTA-treated peripheral blood using salt precipitation method. aCGH using Agilent Technologies $8 \times 60 \mathrm{~K}$ and $4 \times 180 \mathrm{~K}$ platforms (Agilent Technologies, Santa Clara, CA, USA) was performed according to the manufacturer's instructions. Data analysis was done using Agilent 

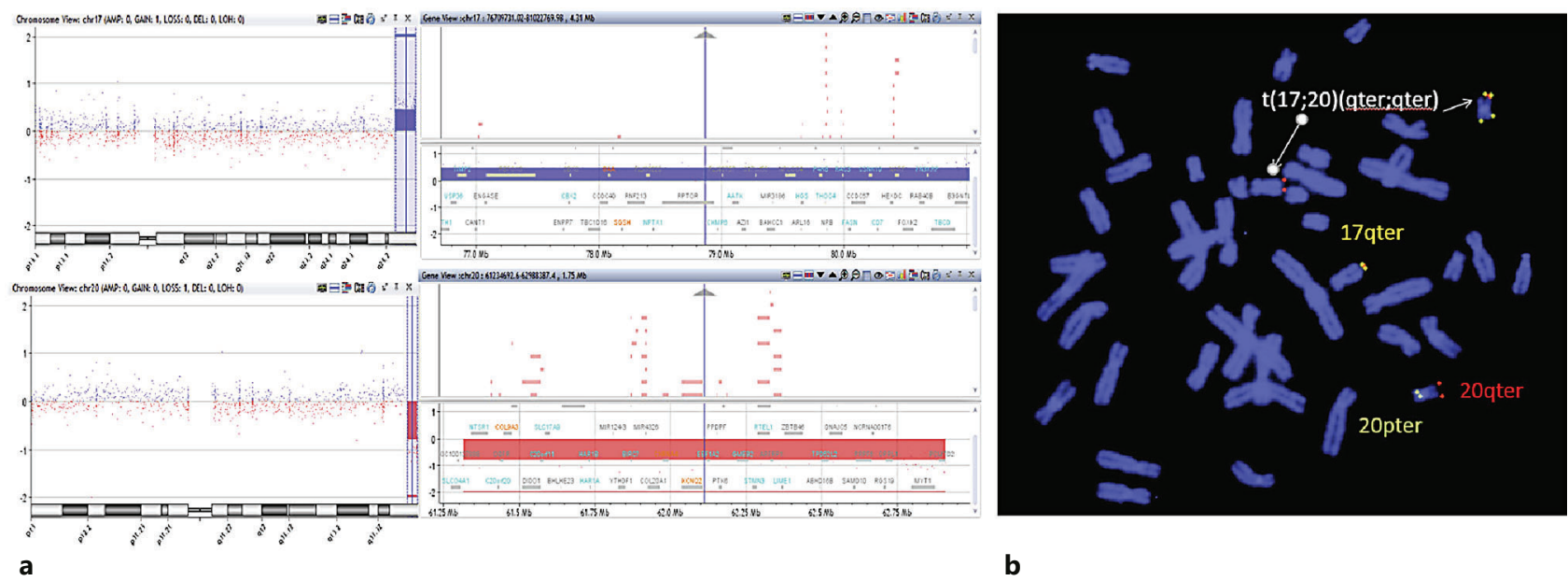

b

Fig. 2. a Microarray analysis of the patients showing partial trisomy $17 \mathrm{q}$ and partial monosomy 20q. Chromosomal view (left) and zoomed-in view (right). The top figure shows the duplication region (blue) and the bottom figure shows the deletion region (red), and the zoomed-in view shows some of the genes in the altered genomic regions. b Subtelomeric FISH for chromosomes 17 and 20 revealed maternal balanced translocations.

scanner and Feature Extraction software. Results were processed by Agilent Cytogenomics software. Detected CNVs were analyzed using in-house data and public databases, such as DECIPHER, DGV, and ClinVar.

\section{Karyotyping and FISH Analysis}

Metaphase chromosome preparations were obtained from phytohemagglutinin-stimulated sodium heparin-treated peripheral blood lymphocyte cultures from the mothers and grandmother according to standard procedures. Chromosomal analyses were carried out on G-banded chromosomes. Subsequently, subtelomeric FISH was performed with Vysis 30-27000 ToTelVysion Probe Kit (17q [Yellow], 20p [Green], 20q [Red]).

\section{Results}

Based on the pedigree showing 3 affected sons from 2 sisters, the inheritance was initially presumed as Xlinked recessive. However, microarray analysis was performed as the first-tier diagnostic tool. aCGH on platform $8 \times 60 \mathrm{~K}$ in patients 1 and 3 revealed a 4,357-kb gain on chromosome 17 (arr[hg19] 17q25.3(76,687,948$81,044,553) \times 3$ ) and $1,594-\mathrm{kb}$ loss on chromosome 20 (arr[hg19] 20q13.33(61,314,406-62,908,674)×1) [ISCN 2016] (Fig. 2). aCGH on platform $4 \times 180 \mathrm{~K}$ in patient 2 showed a duplication of $4,429 \mathrm{~kb}$ on chromosome 17 (arr[hg19] 17q25.3(76,615,819-81,044,553) $\times 3)$ and a deletion of $1,635 \mathrm{~kb}$ on chromosome 20 ( $\operatorname{arr}[\mathrm{hg} 19]$

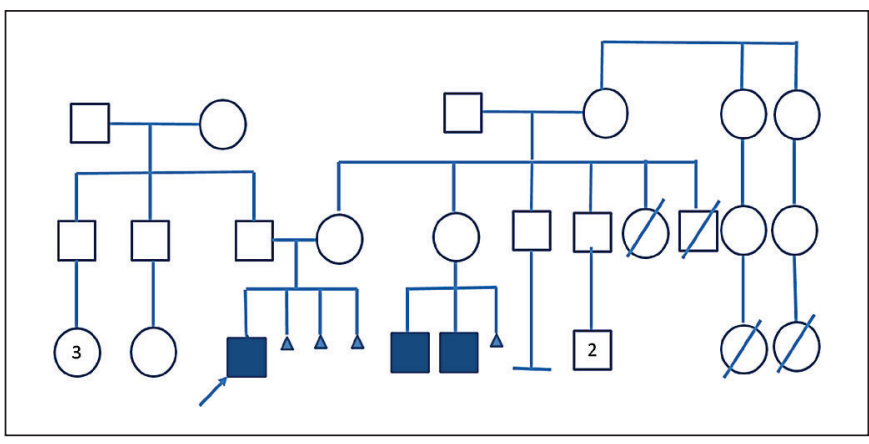

Fig. 3. The pedigree of the family suggestive of maternal inheritance. The arrow indicates patient 1.

$20 q 13.33(61,314,406-62,949,149) \times 1)$. Genomic regions harboring CNVs of 17q25.3 and 20q13.3 encompassed 75 and 41 OMIM genes, respectively. No genomic imbalances were detected in healthy parents with aCGH. The pedigree was highly suggestive of maternal inheritance (Fig. 3). However, maternal karyotypes did not reveal such small chromosomal rearrangements; therefore, subtelomeric FISH for chromosomes 17 and 20 was performed. The assay confirmed the presence of maternal balanced translocations $t(17$; 20)(q25;q13.3) in both sisters, inherited from their mother (Fig. 2).
40

Mol Syndromol 2020;11:38-42 DOI: $10.1159 / 000505141$
Ürel-Demir et al. 


\section{Discussion}

The present study describes 3 individuals from a family, with partial trisomy $17 \mathrm{q}$ and partial monosomy $20 \mathrm{q}$ caused by unbalanced segregation of balanced maternal translocations.

Microduplications of distal 17q, either de novo or inherited, were previously described with various breakpoints. Phenotypic presentation varied depending on the size of the region and the genes involved. In around $85 \%$ of the patients, the condition resulted from unbalanced segregation of parental balanced reciprocal translocations with concomitant partial monosomies [Upadia et al., 2018]. Patients often exhibited DD, ID, short stature, microcephaly, facial dysmorphism, cleft palate, and skeletal anomalies, including rhizomelia, polydactyly, syndactyly, scoliosis, and joint laxity [Upadia et al., 2018]. The craniofacial phenotype showed frontal bossing with bitemporal narrowing, widow's peak, hypertelorism, downslanting palpebral fissures, depressed and broad nasal bridge, long philtrum, thin upper lip, wide mouth with downturned corners, micrognathia, low-set and posteriorly rotated ears, short and a webbed neck with low posterior hairline [Upadia et al., 2018]. Urogenital, cardiac, ophthalmological and central nervous system abnormalities as well as sensorineural deafness were reported [Upadia et al., 2018]. Pure 17q25.3 duplication affected a 3-year-old with DD, dysmorphic features, and arthrogryposis; thus, the authors suggested $17 \mathrm{q} 25.3 \mathrm{qter}$ as the critical region [Lukusa and Fryns, 2010]. The patients in this study shared features of distal $17 \mathrm{q}$ trisomy, such as ID, microcephaly, short stature, facial dysmorphism, cryptorchidism, and joint laxity.

Deletions in chromosome $20 \mathrm{q}$ are characterized by ID, dysmorphic features, limb malformations, growth retardation, behavior problems, and seizures [Traylor et al., 2010]. However, patients with $20 \mathrm{q}$ terminal deletions seem not to exhibit unique phenotypic traits allowing clinical recognition [Traylor et al., 2010; Okumura et al., 2015]. Rather, the deletions were associated with increased seizure risk, particularly those encompassing the KCNQ2 and CHRNA4 genes [Kurahashi et al., 2009; Pascual et al., 2013]. KCNQ2 (MIM 602235) encodes a voltage-gated potassium channel and has been linked to benign familial neonatal seizures (BFNS) and early infantile epileptic encephalopathy. CHRNA4 (MIM 118504) encodes the a 4 subunit of neuronal nicotinic acetylcholine receptors that act as ligand-gated ion channels transducing signals in response to acetylcholine. Mutations in CHRNA4 cause autosomal dominant nocturnal frontal lobe epilepsy (ADNFLE). Kurahashi et al. [2009] stated that concomitant deletions of KCNQ2 and CHRNA4 cause the same phenotype as in deletions of KCNQ2 only, and this phenotype was BFNS instead of ADNFLE. Additionally, Okumura et al. [2015] deduced that 20q13.33 deletions encompassing both genes have more links with BFNS and a milder clinical course.

On the other hand, Pascual et al. [2013] described 3 patients with contiguous KCNQ2 and CHRNA4 deletions and distinguished these from patients with typical BFNS by the presence of DD and lack of epileptic remission. Furthermore, Béri-Deixheimer et al. [2007] argued that 20q13.33 deletions involving CHRNA4 and ARFGAP1 (MIM 608377) may correlate with a relatively severe clinical phenotype, emphasizing the negative effect of haploinsufficiency of these genes. Conversely, several reports speculated that complete deletions of CHRNA4 may not be related to epilepsy development [Kurahashi et al., 2009; Okumura et al., 2015]. This suggests that gain-of-function mutations in CHRNA4 are likely to be implicated in the pathogenesis of ADNFLE [Bertrand et al., 2002]. Each of the present patients experienced their initial seizures during the neonatal period, and only seizures of patient 1 disappeared without treatment. Our observations further support Pascual et al. [2013] in that concomitant KCNQ2 and CHRNA4 deletions present with a clinical phenotype different from typical BFNS.

The deleted regions included several other OMIM genes. Heterozygous mutations in COL9A3 (MIM 120270) cause multiple epiphyseal dysplasia; however, deletions seem irrelevant [Traylor et al., 2010]. Heterozygous and homozygous mutations in SOX18 (MIM 601618) are associated with hypotrichosis, lymphedema, telangiectasia, and renal defects, while deletions are not causative. Mutations in SLC17A9 (MIM 612107), RTEL1 (MIM 608833), DNAJC5 (MIM 611203), and PRPF6 (MIM 613979) lead to other clinical phenotypes, which are absent in our patients. However, heterozygous disruptions of EEF1A2 (MIM 602959) have been detected in patients with severe ID, autism, and epileptic encephalopathy; MYT1 (MIM 600379) encoding a transcription factor highly expressed in oligodendrocyte progenitors, possibly contributes to the neurological impairment [Kroepfl et al., 2008].

To the best of our knowledge, there is only 1 patient previously reported with both partial trisomy $17 \mathrm{q}$ and partial monosomy $20 \mathrm{q}$ resulting from unbalanced translocation [Marques et al., 2015]. Among phenotypic characteristics of that patient, including DD, craniosynosto- 
sis, and congenital heart defects, we consider craniosynostosis is worth noting, being absent in all 3 of our patients. Furthermore, in our patients, copy number aberrations involve larger segments than in the patient described by Marques et al. [2015], considering that craniosynostosis is not an essential feature caused by the disrupted genes in the breakpoint regions. Craniosynostosis has been documented in individuals with $17 \mathrm{q}$ duplication previously, and gain of function in the ACTG1 gene was argued as responsible for premature fusion of cranial sutures. However, many patients with duplication of ACTG1 without craniosynostosis have also been reported, and Marques et al. [2015] proposed that craniosynostosis developed secondarily in their patient.

This report emphasizes the importance of microarrays as the first-tier diagnostic tool in ID, even when the pedigree suggests an apparent X-linked inheritance. Rare chromosomal imbalances, particularly when they are small in size, are difficult to recognize clinically and diagnose microscopically, and therefore should be initially screened using microarrays.

\section{Statement of Ethics}

This study was performed in accordance with the Declaration of Helsinki Principles. Written informed consent was obtained from the families.

\section{Disclosure Statement}

The authors have no conflicts of interest to declare.

\section{Author Contributions}

G.E. Utine and P.Ö. Şimşek-Kiper constructed the idea for research and the manuscript. G.E. Utine, P.Ö. Şimşek-Kiper, and G. Ürel-Demir designed the study. K. Boduroğlu and M. Alikaşifoğlu supervised the course of the project and provided support. G. ÜrelDemir and Ö. Akgün-Doğan collected data. N. Güleray-Lafcı and S. Oğuz carried out experiments. P.Ö. Şimşek-Kiper, G. ÜrelDemir, and G.E. Utine analyzed and interpreted the results. P.Ö. Şimşek-Kiper, G. Ürel-Demir, and G.E. Utine were responsible for literature review. G. Ürel-Demir and Ö. Akgün-Doğan took the lead in writing the manuscript. G.E. Utine, P.Ö. Şimşek-Kiper, and K. Boduroğlu revised the manuscript and finalized it.

\section{References}

Béri-Deixheimer M, Gregoire MJ, Toutain A, Kroepfl T, Petek E, Schwarzbraun T, Kroisel PM, Brochet K, Briault S, et al: Genotype-phenotype correlations to aid in the prognosis of individuals with uncommon 20 q13.33 subtelomere deletions: a collaborative study on behalf of the "association des Cytogeneticiens de langue Francaise”. Eur J Hum Genet 15:446452 (2007).

Bertrand D, Picard F, Le Hellard S, Weiland S, Favre I, et al: How mutations in the nAChRs can cause ADNFLE epilepsy. Epilepsia 43:Suppl 5 112-122 (2002).

ISCN 2016: An International System for Human Cytogenomic Nomenclature; McGowan-Jordan J, Simons A, Schmid M (eds). Cytogenet Genome Res 149:1-140 (2016).
Plecko B: Mental retardation in a girl with a subtelomeric deletion on chromosome $20 \mathrm{q}$ and complete deletion of the myelin transcription factor 1 gene (MYT1). Clin Genet 73:492-495 (2008).

Kurahashi H, Wang JW, Ishii A, Kojima T, Wakai $\mathrm{S}$, et al: Deletions involving both KCNQ2 and CHRNA4 present with benign familial neonatal seizures. Neurology 73:1214-1217 (2009).

Lukusa T, Fryns JP: Pure de novo 17q25.3 micro duplication characterized by micro array $\mathrm{CGH}$ in a dysmorphic infant with growth retardation, developmental delay and distal arthrogryposis. Genet Couns 21:25-34 (2010).

-Marques F, Heredia R, de Oliveira C, Cardoso MT, Mazzeu J, Pogue R: Partial trisomy $17 \mathrm{q}$ and partial monosomy $20 \mathrm{q}$ in a boy with craniosynostosis. Am J Med Genet A 167A:412416 (2015).
Okumura A, Atsushi I, Shimojima K, Kurahashi $\mathrm{H}$, Yoshitomi S, et al: Phenotypes of children with 20q13.3 microdeletion affecting KCNQ2 and CHRNA4. Epileptic Disord 17:165-171 (2015).

Pascual FT, Wierenga KJ, Ng YT: Contiguous deletion of KCNQ2 and CHRNA4 may cause a different disorder from benign familial neonatal seizures. Epilepsy Behav Case Rep 1:3538 (2013).

Traylor RN, Bruno DL, Burgess T, Wildin R, Spencer A, et al: A genotype-first approach for the molecular and clinical characterization of uncommon de novo microdeletion of 20q13.33. PloS One 5:e12462 (2010).

Upadia J, Philips JB 3rd, Robin NH, Lose EJ, Mikhail FM: A case report of chromosome 17q22-qter trisomy with distinct clinical presentation and review of the literature. Clin Case Rep 6:612-616 (2018). 